Alterstice
Revue internationale de la recherche interculturelle
International Journal of Intercultural Research
Revista International de la Investigacion Intercultural

\title{
L'interculturel dans les interactions des professionnels avec les usagers migrants
}

\section{Margalit Cohen-Emerique}

Volume 1, numéro 1, 2011

Penser la recherche interculturelle : le défi des diversités

URI : https://id.erudit.org/iderudit/1077587ar

DOI : https://doi.org/10.7202/1077587ar

Aller au sommaire du numéro

Éditeur(s)

Alterstice

ISSN

1923-919X (numérique)

Découvrir la revue

Citer cet article

Cohen-Emerique, M. (2011). L'interculturel dans les interactions des professionnels avec les usagers migrants. Alterstice, 1(1), 9-18.

https://doi.org/10.7202/1077587ar

\section{Résumé de l'article}

Cet article reprend, avec l'aimable autorisation de l'auteure et de l'éditeur, la presque totalité du chapitre 8 du nouveau livre de Margalit Cohen-Emerique, Pour une approche interculturelle en travail social, théories et pratiques, paru aux éditions des Presses de l'EHESP. L'auteure, chercheure et formatrice en France, dans de nombreux pays européens et au Québec, expose d'abord les origines et les usages du terme interculturel dans une perspective psychosociologique. Selon ses sources, la notion s'applique aussi bien à la recherche comparative qu'à la recherche sur les contacts entre cultures. Les sciences sociales ne sont pas les seules à employer ce terme : il peut aussi qualifier une des nombreuses modalités du " vivre ensemble ». Margalit Cohen-Emerique fait ensuite un rappel des réflexions épistémologiques de Martine Abdallah-Pretceil, qui pose trois perspectives pour marquer l'originalité d'une approche interculturelle et non uniquement culturelle : les perspectives subjectiviste, interactionniste et situationnelle. Cet étayage épistémologique permet à l'auteure de procéder à une analyse des interactions entre professionnels du travail social et usagers culturellement différents. Ces interactions constituent une dynamique identitaire pouvant évoluer vers une menace tant à l'identité de l'usager migrant qu'à celle du praticien. Ce dernier peut mobiliser des réactions défensives pour sortir de la déstabilisation engendrée par la menace, sans pour cela modifier sa perception de la situation. Cependant, il peut aussi mettre en oeuvre des ressources faisant appel à diverses compétences, mais toujours en relation avec un contexte comme avec les enjeux de l'interaction et les statuts des acteurs en présence. Ces ressources le conduisent à un changement de regard sur le demandeur migrant et/ou sa famille et à adopter des attitudes et des comportements favorables au respect de la diversité. En dernier lieu, la notion d'interculturel est appliquée aux pratiques sociales, selon une conception développée par l'auteure au fil des ans.
Ce document est protégé par la loi sur le droit d'auteur. L'utilisation des services d’Érudit (y compris la reproduction) est assujettie à sa politique d'utilisation que vous pouvez consulter en ligne.

https://apropos.erudit.org/fr/usagers/politique-dutilisation/ 


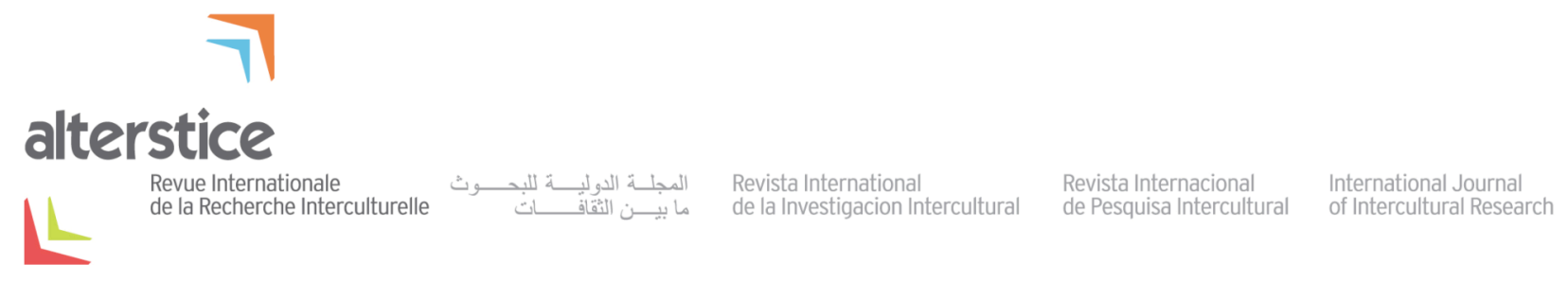

ARTICLE THÉMATIQUE

\title{
L'interculturel dans les interactions des professionnels avec les usagers migrants
}

\author{
Margalit Cohen-Emerique ${ }^{1}$
}

\section{Résumé}

Cet article reprend, avec l'aimable autorisation de l'auteure et de l'éditeur, la presque totalité du chapitre 8 du nouveau livre de Margalit Cohen-Emerique, Pour une approche interculturelle en travail social, théories et pratiques, paru aux éditions des Presses de I'EHESP. L'auteure, chercheure et formatrice en France, dans de nombreux pays européens et au Québec, expose d'abord les origines et les usages du terme interculturel dans une perspective psychosociologique. Selon ses sources, la notion s'applique aussi bien à la recherche comparative qu'à la recherche sur les contacts entre cultures. Les sciences sociales ne sont pas les seules à employer ce terme : il peut aussi qualifier une des nombreuses modalités du « vivre ensemble ». Margalit Cohen-Emerique fait ensuite un rappel des réflexions épistémologiques de Martine Abdallah-Pretceil, qui pose trois perspectives pour marquer l'originalité d'une approche interculturelle et non uniquement culturelle : les perspectives subjectiviste, interactionniste et situationnelle. Cet étayage épistémologique permet à l'auteure de procéder à une analyse des interactions entre professionnels du travail social et usagers culturellement différents. Ces interactions constituent une dynamique identitaire pouvant évoluer vers une menace tant à l'identité de l'usager migrant qu'à celle du praticien. Ce dernier peut mobiliser des réactions défensives pour sortir de la déstabilisation engendrée par la menace, sans pour cela modifier sa perception de la situation. Cependant, il peut aussi mettre en œuvre des ressources faisant appel à diverses compétences, mais toujours en relation avec un contexte comme avec les enjeux de l'interaction et les statuts des acteurs en présence. Ces ressources le conduisent à un changement de regard sur le demandeur migrant et/ou sa famille et à adopter des attitudes et des comportements favorables au respect de la diversité. En dernier lieu, la notion d'interculturel est appliquée aux pratiques sociales, selon une conception développée par l’auteure au fil des ans.

\section{Rattachement de l'auteure \\ ${ }^{1}$ cheurcheure et formatrice indépendante}

\section{Correspondance}

cohen-emerique@wanadoo.fr

\section{Mots clés}

épistémologie; formation professionnelle; intervention interculturelle

\section{Pour citer cet article}

Cohen-Emerique, M. (2011). L'interculturel dans les interactions des professionnels avec les usagers migrants. Alterstice, 1(1), 9-18. 


\section{[...]}

\section{Qu'entend-on par interculturel?}

Le concept est apparu dans les années 1970 aux États-Unis, sous forme d'adjectif ou de substantif, "l'interculturalité ", puis est repris en 1980 par I'UNESCO (Bazin, 1980) et par le Conseil de l'Europe et l'Union européenne. Les rapports de ces deux institutions évoquent successivement : " la formation des enseignants à une éducation pour "la compréhension interculturelle", la nécessité d’aborder les migrations selon une "approche interculturelle", d'inclure "la dimension interculturelle", ou encore de favoriser "l'éducation et la formation interculturelles" » (Dehalu, 2006, p. 24).

Avant d'entrer plus en détail dans le sens que va prendre ce concept dans les pratiques professionnelles ainsi que dans ses modalités d'application, il est nécessaire de donner des précisions sur les significations que lui attribuent les sciences sociales. Ce terme est en effet polysémique et son utilisation imprécise peut prêter à confusion: il désigne à la fois un champ de recherches en sciences de l'homme et de la société et une conception politicoidéologique des modalités de coexistence de la diversité culturelle au sein d’une même société.

\section{L'interculturel en tant que champ de recherches}

Selon la définition de l'UNESCO (Bazin, 1980), il porte sur trois grandes thématiques d'études, complémentaires entre elles :

- l'étude de la diversité culturelle afin de comprendre les sociétés et les individus issus de groupes sociaux et ethniques différents. Dans le cadre de la psychologie, ce champ recouvre les travaux qui cherchent à dégager l'influence de la culture sur les conduites humaines, à tous les niveaux, et dont l'objectif final est de faire progresser les savoirs sur ce qui est de l'ordre du culturel, du social et du psychologique et de leur articulation. Toutes les recherches de psychologie cross-culturelle ou (inter)culturelle comparative sont intégrées dans ce champ;

- les phénomènes liés aux changements culturels, sociaux et psychologiques lors de contacts entre groupes de cultures différentes. Les très nombreux travaux sur les processus d'acculturation de populations en situation de colonisation, d'occupation ou de mondialisation en sont une illustration, ainsi que les études sur les changements culturels chez les migrants. Des approches plus contemporaines se sont orientées sur l'identité sociale et ses transformations en situation de contact avec d'autres cultures, en fonction des groupes en présence et de leur statut respectif;

- les processus d'interactions entre individus et groupes relevant de différents enracinements culturels ainsi que les conditions d'existence des sociétés multiculturelles. Tous les travaux sur les préjugés et les stéréotypes ethniques, sur la discrimination, le racisme, ainsi que toutes les études sur la communication ou la pédagogie interculturelle, sur les relations intercommunautaires dans les quartiers et sur le management interculturel se rattachent à ce champ de recherche. [Toute approche] qui se situe dans ce champ d'études ne peut ignorer les deux premiers.

\section{L'interculturel, clé d'une coexistence réussie?}

L'interculturel est une des conceptions sociopolitiques relatives à la gestion de la diversité culturelle au sein d'une même société qui prévalent dans les pays occidentaux. Chacune de ces conceptions s'est élaborée au cours de l'histoire de la société qui la met en pratique et reflète les courants de pensée et les idéologies qui ont contribué à sa construction. Au niveau individuel, chaque personne se forge sa propre opinion, plus ou moins en accord avec la conception prévalente dans sa société d'appartenance. Elle influencera ses engagements et ses conduites.

On peut recenser plusieurs idéologies dont les points de vue divergent et même s'affrontent: l'assimilation, l’intégration, la lutte contre la discrimination, le multiculturalisme et l'interculturel.

[Dans le texte original sont développées ici les conceptions sociopolitiques d'assimilation, d'intégration et de lutte contre la discrimination. Pour des raisons éditoriales, cette partie ne peut être présentée dans son intégralité.] 
Le multiculturalisme. Conception en vigueur aux Pays-Bas, au Royaume-Uni et au Canada anglophone (le Québec se situerait entre l'intégration à la française et le multiculturalisme), le multiculturalisme, en reconnaissant la multiculturalité des sociétés occidentales et en partant du principe que les individus et la société s'enrichissent de la diversité des cultures en son sein, prône la coexistence de communautés culturelles différentes avec la liberté pour chacune de maintenir sa culture. C'est l'appartenance à une communauté qui prime, au nom de la reconnaissance nécessaire des différentes minorités ethniques. C'est en cela qu'elle se différencie de la conception française qui ne reconnaît pas les minorités en s'appuyant sur le principe de l'égalité de tous devant la loi.

Le multiculturalisme s'accompagne de mesures de lutte contre la discrimination soit sur le plan législatif, soit par une politique de quotas pour que les membres des groupes minoritaires puissent - en fonction de leur représentativité dans la société globale - accéder aux filières scolaires, universitaires et être embauchés dans des cadres de travail où ils courraient sans cela le risque d'être discriminés. C'est ce qu'on nomme «la discrimination positive $"$.

La France, reconnaissant uniquement la communauté des citoyens, rejette le multiculturalisme et les mesures en faveur des minorités à plusieurs titres:

- il conduit à la création de ghettos où chacun reste enfermé dans son milieu et ne s'intègre pas à la société, d'où le danger de communautarisme;

- de plus, les quotas sont considérés comme source de sentiments d'injustice et de discrimination chez les majoritaires;

- enfin, cette conception enferme les individus dans des appartenances où ils peuvent ne pas se reconnaître ou même auxquelles ils n'adhèrent pas.

L'interculturel. Cette conception part de l'idée que la tolérance et le respect à l'égard des individus et des groupes de cultures différentes ne peuvent exister que par une meilleure connaissance mutuelle. Pour cela il ne suffit pas de reconnaître les minorités, il faut créer des espaces de rencontre, de dialogue, de négociation qui rendront possible le respect et la reconnaissance d'individus ou de groupes porteurs d'héritages différents, conditions qui favoriseront l'émergence de "représentations co-construites " (Pagé, 1991), c'est-à-dire des conceptions, des valeurs et des pratiques partagées par tous, produits d'une expérience de vie commune. L'interculturel implique que le mode de pensée occidental n'est pas le modèle culturel supérieur et qu'il ne peut que s'enrichir de la diversité : le groupe dominant doit accepter de ne plus être le seul à organiser la vie sociale et que d'autres modes de vie sont possibles et légitimes à ses côtés, sans toutefois menacer l’identité nationale.

En France, dans les années 1980-1990, tout un courant de chercheurs et d'acteurs de terrain ont prôné cette position; nombreux ont été et sont encore les programmes et les initiatives créatrices qui organisent des rencontres, des échanges, des dialogues entre les "établis » et les immigrés, que ce soit au niveau d'un quartier, d'une mairie, d'une école ou d'une association, dans le but de forger une autre façon de vivre ensemble. Actuellement ce courant s'est affaibli à la suite de changements de politique intérieure et d'événements sur la scène internationale qui ont ravivé la peur du communautarisme.

Un certain nombre de précautions sont à prendre pour éviter les échecs dans la mise en œuvre de cette conception :

- les rencontres et autres échanges doivent être bien organisés, et préparés pour ne pas créer de malentendus et éviter un fiasco qui ne ferait que renforcer les préjugés mutuels;

- il faut trouver des voies pour que les personnes communiquent vraiment et ne restent pas groupées par communauté : sous forme d'une véritable coopération de tous, migrants et travailleurs sociaux, dans la recherche d'objectifs communs. On peut citer en exemple le programme de l'Association des collectifs enfants-parents-professionnels (ACEPP) qui a mis en place des formations, des groupes de réflexion, des expériences pilotes, pour développer, dans ses crèches, une pratique interculturelle fondée sur une véritable prise en compte de la diversité et sur une coopération avec les parents de différentes origines ethniques et sociales. Les résultats sont très intéressants (Cadart, 2006 ; ACEPP, 2008);

- cette conception est très sensible au moindre événement proche ou lointain dans l'espace et le temps qui réactualise la discrimination, le fanatisme, les actes de violence intercommunautaires. Tous les efforts investis 
pendant des années pour rapprocher des groupes ou des individus peuvent alors s'effondrer en un laps de temps très court.

En dépit de ces limites, l'interculturel est une voie intéressante à condition qu'elle soit soutenue par les politiques, particulièrement pour sa mise en œuvre dans le domaine des pratiques sociales qui, par ailleurs, prennent aussi en compte la conception « officielle » de l’intégration.

En dépit de leurs divergences, et face au constat de leurs manques et limites respectives, ces diverses conceptions tendent à se rapprocher. Ainsi, malgré ses principes fondateurs, la France, confrontée à la création de ghettos et aux inégalités marquantes qui caractérisent les populations immigrées, s'oriente à petits pas vers une politique de mixité sociale et vers des mesures qui facilitent l'accès à des cursus auxquels, sans cette aide, les migrants et leurs enfants ne pourraient jamais accéder. De leur côté, les pays anglo-saxons et ceux du nord de l'Europe constatent l'enfermement total de certaines communautés ainsi que les effets boomerang de la discrimination positive auprès de la population dite non discriminée. Aussi cherchent-ils actuellement à pallier ces effets indésirables par des voies diverses, pour préserver l'unité de la société. À la lumière de ses propres expériences, chacune de ces conceptions est amenée à assouplir ses principes idéologiques et à chercher, auprès des autres, des voies différentes tout en les adaptant à son histoire et à son identité.

Ce très rapide aperçu théorique sur le champ de l'interculturel montre que, dans le domaine de la recherche, il reste encore un très long chemin à parcourir pour avoir une idée de la place de la culture dans les sciences de l'homme et de la société. Sa déclinaison dans les pratiques en tant que clé pour apprendre à "vivre ensemble ", reste à préciser et perfectionner. Mais « la vocation des chercheurs [n']est[-elle pas] d'apporter leur contribution à la réflexion sur les moyens de concilier le respect des personnes et l'intégration sociale pour que les sociétés démocratiques puissent continuer d'exister sans s'aliéner dans le désordre » (Schnapper, $2007: 26)$ ?

Cet état des lieux de l'interculturel serait incomplet si nous ne remontions pas à ses fondements épistémologiques.

\section{Approche épistémologique de l'interculturel}

Martine Abdallah-Pretceille (1986) a fait une analyse épistémologique de ce concept qui a éclairé ma démarche de recherche-action. Elle pose que "l'interculturel " apporte, à une approche qui serait seulement "culturelle ", trois perspectives nouvelles : subjectiviste, interactionniste et situationnelle.

\section{La perspective subjectiviste}

Le qualificatif « subjectiviste » implique une relation entre deux individus porteurs de culture différente, chacun se l'étant appropriée dans sa subjectivité et de façon unique après l'avoir reçue, nuancée, modifiée en fonction de son âge, de son genre, de ses appartenances sociales et de sa trajectoire personnelle avant de la transmettre à son tour. On ne rencontre pas une culture, en particulier dans la relation avec des migrants qui sont coupés de leur environnement matériel et social d'origine, mais un individu ou des groupes qui mettent en scène leur culture.

Subjectiviste aussi, car c'est cette culture "subjective » ou " intériorisée » - pas toujours consciente, véhiculée par les entendements individuels et ne s'exprimant que par l'intermédiaire d'individus - qui est confrontée à une autre culture subjective et non conscientisée portée par d'autres individus.

Subjectiviste enfin, du fait que la perspective interculturelle nécessite un travail du sujet-acteur ou du chercheur pour tendre vers une neutralité culturelle par rapport à ses références propres, neutralité à prendre ici non comme synonyme de négation de son identité, mais comme sa reconnaissance " maîtrisée ».

Pour Abdallah-Pretceille, la dimension subjectiviste répond à une triple exigence :

1. éviter toute démarche " classificatrice, simplificatrice et globalisante » qui, comme nous l'avons vu, constitue un obstacle à l'approche de la diversité; 
2. introduire de la souplesse, de la prudence, de la nuance dans le discours, en multipliant les perspectives et les points de vue et en aidant à se défaire de ses préjugés ou autres a priori, et à relativiser ses modèles et ses savoirs;

3. « reconnaître que tout acte, qu'il soit de nature cognitive, relationnelle, affective, pratique ou éducative, s'inscrit dans un réseau d'intersubjectivités " (p.31) ce qui implique, dans la rencontre avec l'autre, de tenter d'appréhender ce qui se déroule "ici et maintenant» en ayant conscience que chacun l'inscrit dans son propre cadre de références culturelles, sociales et personnelles.

\section{La perspective interactionniste}

L'interculturel implique de reconnaître qu'il y a deux acteurs en présence, moi et autrui et non un seul : l'étranger, le migrant. "Toute mise en question de l'autre ne peut qu'être doublée d'un questionnement sur le moi [...]. Méthodologiquement, l'accent doit être mis beaucoup plus sur le rapport que le "je" (individuel ou collectif) entretient avec autrui que sur autrui proprement dit. C'est ce processus de relation en miroir qui fonde le discours interculturel [...]. Cette perspective interactionniste redéfinit la différence, non comme une donnée naturelle, ni comme un fait objectif à caractère statistique et érigé de façon absolue, mais comme un rapport dynamique entre deux identités » (Abdallah-Pretceille, 1986. p. 32).

En d'autres termes, l'autre, par sa différence, joue le rôle de révélateur de ce que je suis. Cette perspective va à l'encontre de l'approche habituelle: expliquer ses difficultés de relation et de communication par la différence culturelle de l'autre, sans prendre en compte qu'elle est toujours relative à soi.

\section{La perspective situationnelle}

Si l'interculturel impliquait seulement des différences de normes, de codes et de valeurs dans l'interaction entre des personnes d'enracinement culturel différent, les difficultés de compréhension seraient relativement faciles à surmonter. Mais les cultures ne se développent pas dans des espaces aseptisés, " elles sont actualisées dans un temps, un lieu marqué par l'histoire, l'économique et le politique » (Abdallah-Pretceille, 1986, p. 32). Elles s'inscrivent dans les hiérarchies qui caractérisent les sociétés humaines et dans les événements qui ont marqué la domination de certains peuples sur d'autres.

C'est pourquoi les interactions interculturelles ne peuvent se dérouler que sur fond de représentations mutuelles forgées dans des contextes précis. Même si les protagonistes de l'interaction n'ont pas été impliqués dans des contentieux qui se situent loin dans le temps ou dans l'espace, ceux-ci ont laissé des traces dans les mémoires collectives et individuelles et sont souvent source d'images négatives, gonflées ou déformées, de réactions de rejet, voire de racisme à l'égard de l'autre. Il y aura toujours une culture jugée supérieure face à une culture jugée inférieure, un pays développé face à un pays sous-développé, un civilisé qui considère l'autre comme un primitif, un ex-colonisé confronté à un ex-colonisateur, un Blanc oppresseur ex-esclavagiste qui rencontre un Noir descendant d'esclave, un majoritaire face à un minoritaire, un menaçant vis-à-vis d'un menacé... Toutes ces dyades, auxquelles peuvent s'ajouter la pauvreté lourde d'une non-reconnaissance radicale ou encore les peurs éveillées par le terrorisme et stigmatisantes pour certaines catégories de population, décrivent une dynamique identitaire basée sur des jeux de pouvoir et des niveaux de valorisation différents entre les protagonistes.

Ainsi, les relations interculturelles sont complexes et difficiles parce qu'elles réveillent le sentiment du " nous » par rapport à " eux ", mais aussi parce qu'elles peuvent raviver des blessures profondes, nées de l'oppression et des persécutions, qui se cicatrisent difficilement car elles sont inscrites dans les identités collectives, transmises de génération en génération et toujours accompagnées d'une mobilisation émotionnelle puissante.

Comme le disait Nelson Mandela, dans son discours d'investiture en Afrique du Sud, le 25 mai 1994 : « Le fardeau du passé pèse sur chacun de nous, ceux qui ont blessé, comme ceux qui ont souffert. " Citons encore Henri Tincq (2001) qui écrit à la suite des attentats du 11 septembre 2001 aux États-Unis : " II y a réactivation chronique d'événements tragiques de deux systèmes d'exclusion mutuelle, Croisades et Djihad qui, depuis le Moyen Âge ont pu opposer l'Islam et l'Occident chrétien et conduit aujourd'hui à tant de malentendus et d'embrasements. ॥ 
Les relations interculturelles, qui sont autant le produit de différences culturelles que de rapports sociaux asymétriques et de contextes historiques dans lesquels elles se déroulent, vont éveiller des processus psychologiques qui peuvent être extériorisés ou au contraire refoulés.

Les processus psychologiques en jeu dans la perspective situationnelle. Quels sont les attitudes et les sentiments qui se manifestent chez les uns, " (ex-)dominants », et les autres, « (ex-)dominés »?

Les descendants des ex-oppresseurs, issus de pays riches et développés, peuvent se sentir coupables ou/et s'autoaccuser, « une tentative plus ou moins hypocrite, en tout cas plus intellectuelle que réelle de tirer son épingle du jeu pour restaurer une innocence illusoire " (Doutreloux, 2000, p. 25), ou encore, sous prétexte de vouloir aider les peuples malheureux ou les migrants, pratiquer un paternalisme derrière lequel se cache souvent un sentiment de supériorité. II y a aussi tous ceux qui déclarent qu'ils connaissent leurs besoins, sans jamais les avoir écoutés, ou encore les repentirs sur le passé de l’opprimé, alliés à un aveuglement total vis-à-vis de son présent et à une défiance sur ses réussites pouvant dégénérer en défaitisme.

Chez les ex-opprimés, peuvent perdurer les sentiments d'avoir été humiliés dans le passé et d'être objet de discrimination dans le présent. Certains développent un conformisme, une soumission, au moins en apparence, visà-vis des majoritaires. D'autres résistent aux pressions à une acculturation trop brutale par un repli communautaire. Chez quelques-uns c'est la suspicion, la méfiance, les reproches massifs qui dominent, pouvant cacher "des ignorances plus ou moins justifiables, des jalousies inavouables ou des bigoteries humanitaires " (Doutreloux, 2000, p. 25). Ou encore sur un registre plus violent, c'est la rancœur, le ressentiment, la haine ou l'enfermement dans la victimisation qui conduisent, ensemble ou isolément - au niveau individuel ou groupal - à construire des théories, des idéologies sur l'altérité, pour atténuer sa frustration et sa souffrance ou pour justifier ses propres conduites qui sont difficilement admissibles.

[...] Le courant de l'ethnicisation décrit chez les migrants cet effet boomerang des attitudes de dévalorisation à leur égard et d'un enfermement dans une appartenance ethnique disqualifiée; c'est un processus qui dénie toute responsabilité propre et culpabilise autrui. Cette attitude dominée par le ressentiment a été décrite par Marc Angenot (1997), comme une tentative de dépasser à moindre coût l'infériorisation, avec des bénéfices immédiats, ne conservant de l'étincelle de révolte et de prise de conscience qu'une dynamique d'animosité, d'autosatisfaction ou « un ayant droit rancunier ». [...]

Ainsi, la rencontre avec l'autre, "ici et maintenant", inscrite dans un contexte politique tendu, peut, des deux côtés, faire de nouvelles blessures ou raviver les anciennes.

Dans les pays d'immigration, en Europe et au Canada, l'intégrisme musulman qui pénètre dans beaucoup de familles émigrées venant de pays du Sud, le maintien chez certaines de pratiques qui vont à l'encontre des valeurs démocratiques des sociétés qui les accueillent (comme les mariages forcés), les attentats perpétrés ou organisés sur leur territoire par des cellules djihadistes, favorisent la montée d'une crispation des deux côtés. Les uns se sentent menacés dans leurs valeurs par tous ces étrangers qu'ils assimilent aux extrémistes, ce qui conduit à la montée en puissance de partis nationalistes. Les autres, la sensibilité à fleur de peau, sont mal à l'aise face à ces amalgames et aux remises en question périodiques de leur intégration. Se joue là une dynamique identitaire où les représentations mutuelles se heurtent, éveillant des affects violents et renforçant des critiques et des attitudes de mise à distance des uns vis-à-vis des autres. [...]

Ainsi, la rencontre entre personnes de cultures différentes va au-delà de la confrontation avec la diversité; c'est une dynamique identitaire où se jouent des contentieux historiques, des rapports asymétriques, des affects profonds, des attitudes et contre-attitudes et des conduites qui peuvent aller jusqu'à la violence, engendrant « une dynamite identitaire ». Bref, on ne peut séparer le culturel de ses dimensions sociale, historique et politique.

\section{L'interculturel dans les interactions entre les acteurs du social et de l'éducatif et les usagers migrants}

L'approche épistémologique de l'interculturel permet de poser la problématique du respect des différences et de ses limites chez les acteurs sociaux, non en référence à un seul porteur de culture, l'autre, le migrant, mais en 
termes de relations et de communication entre deux porteurs de culture, pris dans un sens très large d'héritage, de mémoire, de systèmes de représentations, d'expériences de vie; dans son intervention, l'acteur social met en scène son système de valeurs, ses normes personnelles et professionnelles. S'y ajoutent les statuts sociaux et juridiques totalement différents des interlocuteurs ainsi que les contextes sociaux et historiques dans lesquels se déroule l'interaction.

L'approche interculturelle cerne le professionnel dans sa globalité, en tant que centre cognitif et affectif et comme foyer d'élaboration de sens, en interaction avec un autrui individuel ou collectif, autre foyer d'élaboration de sens. Envisager l'interculturel en ces termes est fondamental :

- d'une part, cela évite de tomber dans le piège très courant de considérer les professionnels comme culturellement neutres, hors des rapports sociaux et sans fonction d'acculturation;

- d'autre part, cette approche permet de mieux comprendre la dynamique identitaire qui caractérise tout processus d'aide, qu'il se déroule dans le cadre d'une action sociale, d'un suivi éducatif ou même d'une aide psychothérapeutique : les rapports de pouvoir, les contentieux historiques comme les échos de l'actualité peuvent être douloureusement présents dans les interactions entre les praticiens et les demandeurs migrants venus de pays au passé lourd de domination.

\section{La dynamique identitaire dans les pratiques professionnelles en travail social et éducatif}

Kalpana Das (1988) analysant les pratiques des travailleurs sociaux au Québec décrit les perceptions mutuelles, exprimées sous forme de notions dyadiques, qu'ont les groupes «blancs » et " non-blancs ». Les premiers utilisent les dyades civilisé/non civilisé, développé/sous-développé, sauveur/sauvé tandis que les " non-blancs » se réfèrent aux couples dominant/dominé, oppresseur/opprimé. "Mais de plus, la perception qu'ont les "non-blancs" d'euxmêmes n'est pas homogène, ils se placent et placent les autres à des niveaux différents de l'échelle supérieur/inférieur, mais tous le font selon les paramètres du "blanc" qu'ils ont intériorisés » (p. 44).

Dans notre recherche-action, un certain nombre d'observations recueillies dans les stages montrent que cette dynamique identitaire n'est pas sans effet dans le face-à-face avec l'usager migrant, le parent ou le jeune issu de l'immigration, soit en amont de la rencontre, soit en aval.

Les acteurs sociaux, dont les valeurs fondamentales reposent sur le respect de la personne quelle que soit sa couleur, son origine ou sa croyance, n'ont pas conscience de cette dynamique, ni par rapport à eux-mêmes, ni concernant l'autre. Aussi sont-ils déroutés par des réactions qui souvent ne peuvent s'expliquer que dans une perspective situationnelle. En voici deux exemples :

Situation 14 : "Es-tu raciste?"

Une assistante sociale raconte: "J'avais un client africain que j'avais rencontré déjà quatre fois; à la fin du cinquième entretien, il me dit en me serrant la main : "Tu sais, je voulais savoir au début si tu étais raciste !" ॥

$\Rightarrow$ Pour l'usager, la relation Blanc/Noir était sous-jacente à la relation d'aide et son élucidation était même le préalable à toute relation de confiance avec l'assistante sociale. Pour elle, sans aucun préjugé vis-à-vis des Noirs, cette « dyade » ne jouait aucun rôle.

\section{Situation 15 : Au secrétariat}

Au cours d'une séance de formation auprès des secrétaires du Service social d'aide aux émigrants (SSAE), qui avaient un rôle important d'accueil, d'information et de mise en confiance de la clientèle migrante, celles-ci se plaignaient de l'agressivité de clients à leur égard.

- L'analyse montrait que les demandeurs arrivaient souvent au service social après une démarche à la préfecture de police pour obtenir un permis de séjour. Ils avaient dû faire des heures de queue pour s'entendre dire à l'arrivée au guichet qu'il leur manquait un papier et qu'ils devaient s'adresser au SSAE pour être guidés. La frustration et la rancœur accumulées par leurs vaines démarches se reportaient sur les secrétaires beaucoup plus compréhensives et accueillantes. 
Nous avons constaté combien le passé lourd d'une histoire commune et conflictuelle entre la France et l'Algérie peut peser sur la relation entre un travailleur social et un émigré ou un enfant d'émigré algérien ou un enfant de harki. Le premier peut investir dans la relation, de la culpabilité ou de la méfiance, les seconds peuvent véhiculer de la rancœur, des frustrations, voire du ressentiment. Malheureusement, les travailleurs sociaux qui n'ont pas connu cette histoire douloureuse de la France ont été aveugles et sourds à ces aspects, en particulier concernant les harkis et enfants de harkis. Depuis leur fuite de l'Algérie vers la France dans les années 1960, ces familles ont été nombreuses à s'adresser aux services sociaux, dans les régions où elles avaient été installées, mais cette dynamique identitaire est totalement passée inaperçue auprès des aidants, ce qui a rendu difficile un véritable processus de soutien. II est vrai que les politiques sociales à l'égard des harkis, qui ne prenaient pas en compte leur besoin de reconnaissance symbolique, n’ont pas facilité le travail des professionnels.

Certains professionnels dont les parents étaient des immigrés venant des pays ex-colonisés portent en eux des prémices de ressentiment liées au manque de reconnaissance, voire au mépris et à l'exploitation que leurs parents - en particulier leur père - ont subis; mais leur propre parcours, en dépit de ce ressenti, n'a aucune valeur éducative pour aider les jeunes à sortir de la victimisation et à se prendre en charge s'ils ne prennent pas de distance par rapport à ce ressentiment. Dans le même ordre d'idées, on peut entendre dire dans les stages par certains praticiens: "Mais pourquoi est-ce toujours à nous de faire des efforts et pourquoi n'exige-t-on rien d'eux? " Cela relève du même processus, celui de se présenter comme objet d'une injustice, pour renforcer sa position de pouvoir.

Nous avons pu aussi observer la rancœur des professionnels québécois envers les immigrés qui n’apprenaient pas le français ou envoyaient leurs enfants à l'école anglophone. Ces choix étaient pour eux un déni de leur identité et une non-reconnaissance de tous les efforts que le pays faisait pour les intégrer.

À la suite des révoltes dans certaines banlieues et de leur stigmatisation, les acteurs de terrain ont exprimé leur frustration et leur colère de voir abandonner ces zones, tout en condamnant l'image négative qui en est diffusée, alors qu'existent beaucoup de réalisations positives individuelles ou associatives. Et comme les médias en parlent soit en considérant les habitants de ces quartiers comme des victimes, soit en accusant les politiques sociales d'avoir créé ces situations de marginalisation, on assiste à une cacophonie concernant le problème des banlieues alors qu'il mériterait une analyse objective par une approche complexe, basée sur des données vérifiées, site par site.

Néanmoins, les dynamiques identitaires en jeu dans le travail social ne sont pas fixées une fois pour toutes, figeant chacun dans sa position de dominant ou dominé : le rapport oscille selon les situations et les représentations de celles-ci.

\section{L'identité professionnelle menacée en situation interculturelle}

Dans l'interaction interculturelle entre travailleurs sociaux et usagers migrants, nous avons étudié la dyade menaçant/menacé. Notre recherche montre en effet que l'intervenant social ou éducatif peut, dans certaines situations, se sentir menacé symboliquement par le demandeur, et ceci malgré son statut de majoritaire et de représentant institutionnel. Il est mal à l’aise, voire ressent de l'anxiété s'il ne se sent pas reconnu ni confirmé dans son identité professionnelle par l'usager migrant, comme dans les quelques situations suivantes:

- lorsque le praticien ne comprend pas la langue parlée par un groupe alors qu'il intervient tout seul. II a l'impression qu'on dit du mal de lui;

- lorsqu'il y a inversion des rapports sociaux, du fait que le minoritaire impose ses codes ou traite le praticien avec hauteur, ce qui peut être fréquent avec les réfugiés politiques qui ont perdu leur statut social;

- lorsque le praticien ne réussit pas à mettre en œuvre les modèles professionnels qui fondent son expertise. C'est toute sa valeur professionnelle qui est ébranlée, toute son identité qui est menacée, inhibant sa capacité à s'adapter, tout au moins momentanément, aux réalités rencontrées.

Cette menace à l'identité du professionnel avec sa cohorte de sentiments d'impuissance, de dévalorisation, suscite de multiples réactions de défense. Celles-ci sont à la fois de l’ordre des mécanismes de défense freudiens - la projection, la rationalisation, le déni... - et de l'ordre des réactions de défense sociale - la réaffirmation de sa 
légitimité et de sa position d'expert, le formalisme ou l'enfermement dans les règlements, l'approche diagnostique psychologisante ou psychiatrisante à l'égard de l'usager, le recours à des préjugés sur lui... - (Cohen-Emerique et Hohl, 2004). Elles servent à défendre la valeur de l'identité professionnelle et à retrouver l'estime de soi et une sécurité interne, mais ne changent en rien la représentation du client menaçant ni ne facilitent la relation avec lui.

Alors qu'on aurait tendance à ne voir basculer la dyade menaçant/menacé que du côté du migrant, l'expérience montre que le statut de supériorité et de menaçant oscille entre le professionnel et l'usager, en fonction des situations et des identités en présence. Dans cette dynamique identitaire, le professionnel peut éprouver un fort sentiment d'impuissance entraînant des blocages dans la relation et dans l'action tandis que le migrant aura des réactions de repli sur soi, méfiance, hostilité et l'aide échouera.

Mais il ne faut pas voir ce positionnement défensif comme systématique, un certain nombre de professionnels ont des ressources, acquises par l'expérience ou en relation avec leur personnalité; elles leur permettent de dépasser la menace et de s'ouvrir à l'autre en lui témoignant un intérêt authentique (Cohen-Emerique et Hohl, 2002).

\section{L'interculturel dans la perspective des pratiques professionnelles}

\section{Définition}

C'est l'interaction de deux identités qui se donnent mutuellement un sens dans un contexte à définir à chaque fois. $C^{\prime}$ est un processus ontologique, d'attribution de sens et de dynamique de confrontation identitaire, qui peut malheureusement évoluer vers un affrontement identitaire, une « dynamite » identitaire [...].

L'expression " attribuer un sens » doit être prise ici dans trois acceptions :

- donner une signification, une interprétation à des comportements qui aille au-delà du schéma stimulusréponse;

- choisir une orientation d'action;

- prendre en compte la dimension corporelle, le mot « sens » renvoyant aussi aux cinq sens.

Ces trois dimensions constituent ensemble une logique invisible qu'il est difficile de déchiffrer quand on n'en possède pas les clés, ce qui engendre des réactions émotionnelles plus ou moins violentes et une incapacité à utiliser ses ressources.

La situation 16 est un exemple d'interaction qui illustre les deux expressions-clés de cette définition, " l'attribution de sens » et la « dynamique identitaire ».

Situation 16 : Une fête de fin d'études au Maroc

Dans le cadre d'une formation initiale d'éducateurs spécialisés organisée par la protection judiciaire de la jeunesse sur le thème de la relation et de la communication interculturelle, un voyage avait été organisé au Maroc. Au retour, une journée de formation avait été consacrée aux expériences de chacun durant ce séjour. Un étudiant raconte que tout le groupe avait été invité à la cérémonie de la remise de diplômes d’une école d'éducateurs à Rabat. Quels n'avaient pas été son étonnement et son malaise à la vue des éducatrices marocaines magnifiquement parées de caftans brodés et de somptueux bijoux, fardées et coiffées comme pour un mariage. Les éducateurs marocains étaient aussi très élégants, en complet veston, cravatés. Lui ne portait qu'un jean et un Tshirt délavés. II n'avait pas emporté de tenue habillée ni même un pantalon de ville et une chemise. Partant dans un pays "sous-développé », il s'était dit qu'il ne fallait pas étaler sa richesse face à la pauvreté. Durant toute la cérémonie, il est resté dans un coin, très mal à l'aise, d'autant plus que ses collègues français, eux, avaient prévu une tenue de sortie.

Dans cette situation, le processus ontologique d'attribution de sens se réfère à l'image du Maroc qu'a le narrateur: pays pauvre où les habitants sont démunis de tout. La dynamique identitaire s'exprime dans sa conception idéologique des relations entre personnes originaires de pays riches et celles de pays pauvres: "il faut que les premiers ne montrent en aucun cas leur supériorité pour ne pas rabaisser les seconds ". Du côté des collègues marocains, on peut seulement se poser des questions : comment ont-ils décodé le choix dans son habillement en 
ce jour de cérémonie? Se sont-ils dit : "il nous méprise » ou " c'est un pauvre type sans le sou qui affiche sa misère sans honte "??? Heureusement que la rencontre des éducateurs français avec leurs homologues marocains a été courte. Si elle avait duré plusieurs jours, on peut faire l'hypothèse que des malentendus entre les deux groupes d'éducateurs auraient pu se développer à partir des représentations biaisées des statuts des uns et des autres.

\section{$[\ldots]$}

\section{Déclinaisons pratiques de l'interculturel}

Pratiquer l'interculturel, c'est émettre des hypothèses, et ne pas s'arrêter à une seule; or on reste trop souvent fixé à sa première hypothèse, et ce d'autant plus qu'on se trouve face à l'incompréhensible, à l'inattendu ou envahi par une idée préconçue.

En situation interculturelle, on ne peut rien analyser ni comprendre si on ne saisit pas la signification des comportements et des situations en tenant compte du point de vue de chacune des deux parties.

Il est essentiel pour les professionnels de suivre ces mêmes voies: analyser le problème vu par les deux parties et/ou faire plusieurs hypothèses. Nous avons souvent observé comment très vite, face à l'incompréhensible, à l'inattendu ou à l'envahissement par une idée préconçue, on s'accroche à une seule hypothèse totalement déconnectée du contexte dans lequel le problème apparaît et sans relation avec les rôles et statuts en présence.

Les relations interculturelles constituent une dynamique identitaire complexe que l'on retrouve chez les travailleurs sociaux, mais dont ils n'ont pas conscience, la logique humanitaire de leurs professions en atténuant les processus. C'est pourquoi il est important que ces acteurs sociaux clarifient leurs propres représentations, réelles ou imaginaires, vis-à-vis de l'étranger en général et/ou de telle ou telle catégorie particulière d'étrangers.

\section{Références bibliographiques}

Abdallah-Pretceille, M. (1986). Pédagogie interculturelle : bilan et expertise. Dans C. Clanet (dir.), L'interculturel en éducation et sciences humaines (p. 25-32). Toulouse : Université de Toulouse-Le Mirail.

Angenot, M. (1997). Les idéologies du ressentiment : essai. Montréal : Éditions XYZ.

Association des collectifs enfants parents professionnels (ACEPP) (2008). Construire une pédagogie à partir de la diversité des enfants et des familles : un enjeu pour la petite enfance. Paris: Publication ACEPP.

Bazin, L. (1980). Les conditions d'objectivité dans l'approche interculturelle : le cas des études orientales et asiatiques. Dans Introduction aux études interculturelles. Esquisse d'un projet pour l'élucidation et la promotion de la communication entre les cultures (p. 66-83). Paris : Unesco.

Cadart, M.-L. (2006). Des parents dans les crèches, utopie ou réalité ? Toulouse : Érès.

Cohen-Emerique, M. et Hohl, J. (2002). Les ressources mobilisées par les professionnels en situation interculturelle. Éducation permanente, 150, 161-195.

Cohen-Emerique, M. et Hohl, J. (2004). Les réactions défensives à la menace identitaire chez les professionnels en situations interculturelles. Cahiers internationaux de psychologie sociale, 61, 21-34.

Das, K. (1988). Travail social et pluralisme culturel au Québec : les enjeux inexplorés. Interculture, 100(21), 31-53.

Dehalu, P. (2006). Quand I’Europe découvre l'interculturel. L'Agenda interculturel, 246, 23-24.

Doutreloux, A. (2000). Continent noir. Montréal : Les Éditions Francine Breton.

Pagé, M. (1991). Conserver le pluralisme ethnoculturel par les institutions démocratiques. Dans M. Lavallée, F. Ouellet et F. Larose (dir.), Identité, culture et changement social (p. 113-125). Paris : L'Harmattan.

Schnapper, D. (2007). Qu'est-ce que l'intégration ? Paris : Gallimard.

Tincq, H. (2001, 2 octobre). L'encre du savant et le sang du martyr. Le Monde. 